\title{
Transforaminal Ventral Epidural Adhesiolysis
}

\author{
Michael Hammer, MD*, Daniel M. Doleys, PhD**, and Ok Yung Chung, MD, MBA ${ }^{\#}$
}

Epidural fibrosis with chronic low back pain, nonresponsive to traditional measures of treatment including surgery, is a common entity in modern medicine. Traditionally, epidural steroid injections have been employed to treat chronic low back pain and radiculopathy associated with failed back surgery. Due to the poor effectiveness of epidural steroid injections in post lumbar laminectomy syndrome, epidural adhesiolysis was introduced in the early 1980s. Caudal epidural adhesiolysis with hypertonic saline neurolysis has been described extensively in the literature and has been proven to be relatively successful and safe. To improve the results and reach the target area with steroid, transforaminal ventral epidural adhesiolysis has been utilized.

This retrospective case analysis included 14 patients. Trans- foraminal ventral epidural adhesiolysis was performed on an outpatient basis in all patients. The results showed 93\% improvement initially, which decreased to $71 \%$ at 1 month, $57 \%$ at 3 months, $43 \%$ at 6 months and $21 \%$ at 1 year.

The results of this case study show that ventral epidural lysis of adhesions with hypertonic saline neurolysis is safe and effective in managing chronic low back and lower extremity pain in patients who failed to respond to other conservative modalities of treatments, including fluoroscopically directed transforaminal epidural steroid injections.

Keywords: Epidural fibrosis, transforaminal ventral epidural adhesiolysis, chronic low back pain
Epidural fibrosis leading to chronic low back pain, nonresponsive to traditional measures of treatment including surgery, is a common entity in modern medicine (1-13). Adhesions of the epidural space have many etiologies, including surgical intervention, disc herniation, internal disc disruption, infection, vertebral body fracture, arachnoiditis, mechanical instability, pseudomeningocele, trauma and myelography-associated subarachnoid hemorrhage. Although considerable debate exists as to whether epidural fibrosis causes pain (14-19), it is widely accepted that scar tissue renders the nerves susceptible to injurious histologic changes $(20,21)$. Scar tissue is generally found in three compartments of the epidural space: lateral, ventral and dorsal $(20,22)$.

From *Comprehensive Pain Center, Birmingham, AL, **Pain and Rehabilitation Institute, Birmingham, AL, and ${ }^{*}$ Vanderbilt Pain Control Center, Nashville, TN. *Dr. Hammer is chief of pain management at St. Vincent's Hospital, **Dr. Doleys is the medical director at the Pain and Rehabilitation Institute, and "Dr. Chung is an interventional pain physician at the Vanderbilt Pain Control Center. Address correspondence: Michael Hammer, MD, Comprehensive Pain Treatment Center, Suite 407, 2700 10th Avenue South, Birmingham, AL 35205.
Traditionally, epidural steroid injections have been employed to treat chronic low back pain and radiculopathy associated with failed back surgery. Although there have been no well-controlled studies to determine the true efficacy of epidural steroid injections, it still remains a firstline treatment (9). Its relative ease of performance, initial pain reduction, safety and comfort have perpetuated its popularity. Among the three approaches available to access the lumbar epidural space, namely intralaminar, transforaminal, and caudal, the transforaminal approach is considered as most target specific in fulfilling the aim of reaching the primary site of pathology (9). Caudal epidural injection of drugs was introduced as the first type of entry into the epidural space in 1901, whereas transforaminal epidural injection was introduced as the first and earliest use of epidural steroids (9, 23, 24). Following the same tradition, caudal epidural adhesiolysis was also introduced early in the 1980s (3, 5-9, 13, 16, 18, 25). The objective of an epidural steroid injection is to deliver corticosteroid close to the site of pathology, presumably onto an inflamed nerve root (26). This philosophy is based on the premise that the corticosteroid delivered into the epidural space yields higher local concentrations over an inflamed nerve root and will be more effective than steroid administered either orally or by intramuscular injection. Target-site concentration of steroids depends upon multiple injection variables, though mainly it is the route of epidural administra- 
tion (26, 27). Caudal and interlaminar lumbar epidural injections are affected by the presence or absence of epidural ligaments or scarring, which may prevent migration of the posteriorly administered injectate to the anterior epidural space. Various disadvantages of caudal and lumbar interlaminar epidural injections have been described (27). Similarly, causes of failure of epidural steroid injections also have been described $(27,28)$. It was also shown in normal volunteers that the transforaminal approach showed good ventral flow, whereas the interlaminar method showed predominantly dorsal flow, which was more removed from the usual site of inflammation (29). In evaluating the clinical effectiveness of interventional techniques in the management of chronic pain, transforaminal epidural steroid injections showed a better effectiveness profile with effectiveness evidence strength presenting as strong/ moderate compared to moderate/limited for interlaminar epidural steroid injections and at the same level as caudal epidural steroids, which were classified as strong (9). Thus, the development of transforaminal ventral epidural adhesiolysis was visualized to produce better results with improved adhesiolysis and decompression, finally leading to target-specific delivery of the injectate.

Surgical approaches to the removal of compressive scar tissue are invasive and costly and do not guarantee success, as there may be regrowth of the scar tissue or irreversible nerve damage $(1,4,30)$. An alternative which can be used in patients with failed spinal surgery is catheter-guided lysis of epidural adhesions. Epidural adhesiolysis or neuroplasty in the posterior epidural space is the treatment most frequently employed $(5-9,13,18$, 25). This entails placing a fluoroscopically guided, spiraltipped catheter via the sacral canal into the "filling defect" found on prior epidurography. The catheter commonly follows a path in the posterior epidural space and the tip is positioned in the scar tissue, laterally, as close to the affected nerve root as possible. This procedure has been described extensively in the literature and has proven to be of relative success and safety for many years $(5-9,13$, 18, 25).

\section{METHODS}

This retrospective case analysis included 14 patients. The inclusion criteria for this technique were failed back syndrome characterized by complaints of back and radicular lower extremity pain, epidural fibrosis secondary to either failed back syndrome or inflammatory irritation from chemical irritation of the disc. Positive "filling" defect on epidurography, presence of scar tissue on magnetic resonance imaging (MRI), and a positive response to transforaminal selective epidural injection were essential prior to undergoing transforaminal lumbar epidural adhesiolysis.

Transforaminal ventral epidural adhesiolysis was performed on an outpatient basis in all patients. An intravenous infusion during mild sedation and appropriate monitoring was carried out in each patient. All procedures were performed in the operating room under sterile technique under fluoroscopic visualization. In order to reach the anterior portion of the nerve, a needle was placed obliquely through the intervertebral foramen. After appropriate positioning of the fluoroscopic image with alignment of vertebral endplates, the fluoroscope was directed in a $35-40^{\circ}$ oblique angle to visualize the opening of the intravertebral foramen. At this time, a 17-guage Touhy blunt trochar was advanced using tunnel vision under the pedicle. Once appropriate contact with bone was made and confirmed under fluoroscopy, the needle was then slowly advanced from the posterior (dorsal) border of the neural foramina to the intravertebral space parallel to and "hugging" the inferior border of the pedicle. Appropriate care was taken to avoid nerve injury and only minimal paresthesia was elicited while advancing to the posterior vertebral body. Once the needle tip was revealed to be in the mid foraminal or subarticular zone with a posterior anterior image, with the needle bevel facing posteriorly a Brevikath ${ }^{\circledR}$ catheter (EpiMed International, Inc., Gloversville, NY) was gently advanced under the exiting nerve root and into the ventral epidural space. Whenever necessary, the bevel was rotated cephalad to steer the catheter appropriately. Following this, after achieving a confirmation of ventral lateral radicular contrast spread, an initial injection of 3.5 $\mathrm{mL}$ of $0.5 \%$ bupivacaine with or without $100 \mathrm{mg}$ of fentanyl followed by 1500 units of hyaluronidase $(1 \mathrm{~mL})$ and slow infusion of $10 \%$ sodium chloride solution with the injection of triamcinolone acetate $40 \mathrm{mg}$ was carried out. Following this, the catheter was removed and patients were discharged home. Figs. 1 to 4 show the transforaminal ventral adhesiolysis technique utilized in these patients.

Each patient was evaluated for the amount of relief of pain on the basis of a verbal 10-point pain scale during each visit. Patients were also evaluated for any potential complications. Demographic features of age, mode of onset of pain, gender, weight, height, duration of pain, and history of previous surgical intervention were recorded. 
An example of the transforaminal neurolysis technique is presented here. The patient previously had a herniated nucleus propulsus at L5-S1 encroaching on the L5 nerve root. Said patient presented with L5 neuropathic symptomatology following laminectomy/microdiscectomy at L5 to S1.

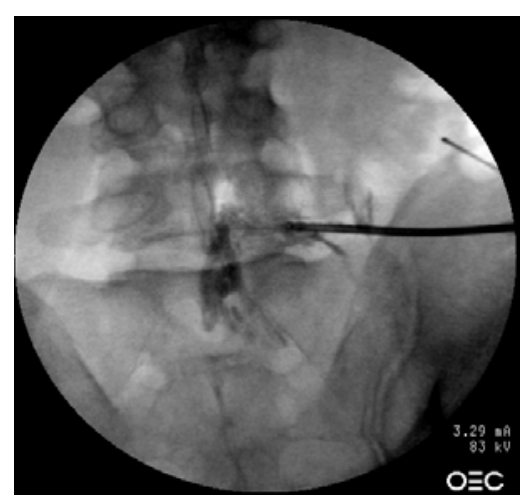

Fig. 1. Needle placement in the lateral recess of the L5 neural foramen. Contrast study shows a filling defect.

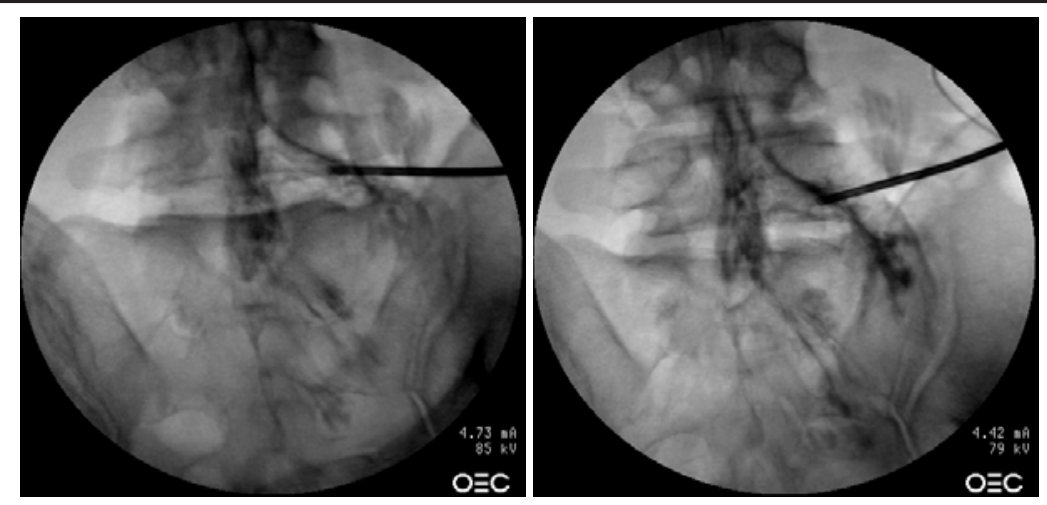

Fig. 3. After hydromechanical dissection/irrigation with 20 сc of sterile saline, a repeat posteroanterior contrast study clearly demonstrates free flow of contrast along the epidural space, exiting nerve root, dorsal root ganglion, and exiting ventral ramus.

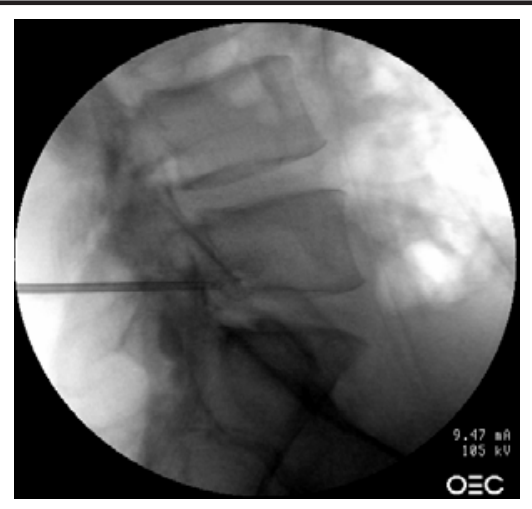

Fig. 4. Final confirmation of 360 degrees' circumferential adhesiolysis is confirmed by a lateral view demonstrating an unobstructed L5 nerve root.

\section{RESULTS}

Demographic data are shown in Table 1. Pain relief is illustrated in Fig. 5. The quality of pain relief was characterized as less than $50 \%$ relief, or greater than $50 \%$ relief. Pain relief greater than $50 \%$ was considered significant, and these patients were characterized as successful with "significant pain relief." As shown in Fig. 5, 13 of 14 patients obtained significant pain relief, however for less than 1 month. At 1 month the significant relief decreased to $71 \%$ of the patients, whereas significant relief decreased to $57 \%$ at 3 months, $43 \%$ at 6 months and $21 \%$ at 1 year.

Patients were evaluated for various types of complications, including infection, rash, and subarachnoid blockade. There were no complications of subarachnoid blockade or infection. In addition, there were no reports of arachnoiditis, paralysis, weakness, bladder disturbances, or any other serious complications. 


\section{Table 1. Patient characteristics}

\begin{tabular}{lcc}
\hline Number of patients & & 14 \\
Gender & Male & $43 \%(6)$ \\
Age (yrs.) & Female & $57 \%(8)$ \\
& Range & $26-59$ \\
Weight (lbs.) & Rean + SEM & $44.9+3.23$ \\
& Mean + SEM & $101-240$ \\
Height (inches) & Range & $63-74$ \\
& Mean + SEM & $68.9+0.92$ \\
Mode of onset of pain & Occupational & $29 \%(4)$ \\
& Nonoccupational & $14 \%(2)$ \\
& Nontraumatic & $57 \%(8)$ \\
Duration of pain (months) & Mean + SEM & $63.8+12.86$ \\
Postlumbar Laminectomy & & $50 \%(7)$ \\
\hline ( ) Number of patients & &
\end{tabular}

\section{DISCUSSION}

Epidural fibrosis is a common phenomenon resulting in chronic intractable low back pain not amenable to traditional modalities of treatment. Epidural fibrosis is detected in lateral, ventral, and dorsal compartments of the epidural space $(20,22)$. In the ventral epidural space, scar tissue can form from the disc and/or the vertebral body (22). A defective disc may leak glycoproteins (31), immunoglobulin-G (32), and phospholipase (33), as well as antigenic substances (34). Continued leakage of these chemical substances may perpetuate an inflammatory response via sensitization of previously silent nociceptors. Scar tissue resulting from defects in the disc, i.e., contents of the nucleus pulposus, may persist despite surgical treatment and continue to produce chronic low back and radicular pain (35). Chronic low back pain may persist as a "mechanical pain" by continued posterior and posterolateral annular fissuring since fibrosis "fixes" the nerve root in one position and increases the susceptibility of the nerve root to tension or compression (36-38).

Histological injury to the nerve roots and dorsal root ganglia may occur with or without compression, resulting in persistence of radicular symptoms (35). Structures in the ventral epidural space may become highly sensitized by mechanical and/or chemical irritation, resulting in axial or

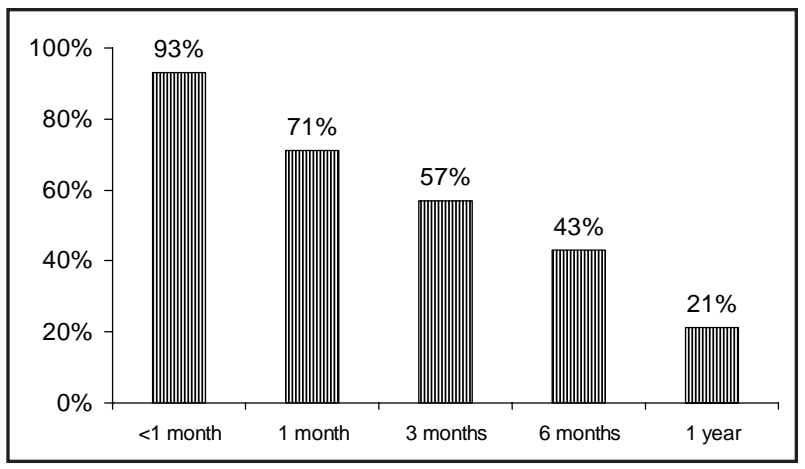

Fig. 5. Illustration of relief $(>50 \%)$ in months following transforaminal ventral epidural adhesiolysis

extremity pain (36). These vulnerable structures include the ventral dura, posterior longitudinal ligament, vertebral periosteum, dural attachments and epiradicular components (nerve-root sleeve or epidural membrane). The ventral epidural space is extensively innervated by the sinuvertebral nerve and sympathetic nervous system and consequently plays an important role in the transmission of nociceptive information $(37,38)$. This complex network of nerves can highly sensitize the area, resulting in chronic low back pain.

Clinically, dorsal root compression presents as a sharp lancinating pain, which is dependent upon movement. Therefore, traction on radicular elements produces radicular pain, i.e., sciatica. Ventral root compression produces a very different sensation. This pain is a deep aching pain, which can be debilitating to the patient. It often involves muscle spasms and cramping.

The anatomy of the lumbar epidural space and foramen has been studied extensively (39-41). Myelography (42), spinal epiduroscopy $(43,44)$, MRI and computed tomographic scan technologies in cadavers (45) have been used. These studies have provided anatomical information on structures of the intervertebral foramen, which include the relative locations of existing neural and vascular elements, along with the fixation of the extrathecal epiradicular structures, nerve roots, and dura. Foraminal dural attachments are more dense and firmly developed at lower vertebral levels such as the L5-S1. These attachments appear to fix the exiting spinal nerves to the ventral root canal and play an important role in the generation of radicular symptoms. Nerve root irritation or traction can result in radicular pain.

Ventral dural attachments also bind the dura between the lateral and the medial foramen and posterior longitudinal ligament, thus contributing to axial pain $(40,41)$. This 
condition exemplifies “neuropathic axial pain.” Low back pain will thus be elicited by straight leg raise testing, as this maneuver produces a "shearing" or tension on the ventral dura. Spencer et al (40) identified ligaments connecting the "sheath of the extrathecal posterior nerve root to the inferior pedicles of the respective foramen." When traction is applied to the posterior longitudinal ligament and vertebral periosteum, the result is low back, hip and/ or buttock pain.

Percutaneous transforaminal epidural adhesiolysis is a novel approach to painful structures in the ventral epidural space. In the traditional transcaudal technique, the catheter often ends up in the dorsal epidural space but rarely can be navigated out into the root canal, thus possibly missing the pathologic lesion. The use of neuroforaminal endoscopy to enhance this transforaminal ventral approach can reveal inflammation in ventral structures. Inflammation and space occupying lesions often prevent precise injection by a posterior approach. The transforaminal approach to lysis of epidural adhesions appears to provide a safe and accurate delivery of medications into an area of the spinal column not previously addressed.

Transforaminal epidural adhesiolysis, like caudal or intralaminar epidural adhesiolysis, imposes a variety of considerations and concerns. Overpressurization (hydraulic decompression/neurolysis) by pressure and/or volume may cause iatrogenic compartment syndrome, producing ischemia of the cauda equina, thus resulting in compression of neural elements and ensuing dysfunction. Slow injection with low pressure on the syringe is essential. Complications from overpressurization may include sexual, bowel, and bladder dysfunction and worsening of pain. There may be a greater potential for harm in the ventral than dorsal area. Due to a rich vascular supply, there is an increased tendency for bleeding and epidural hematoma, damage to dorsal root ganglia via compression, ischemia, etc.

General precautions involving this technique include the usual concerns when applying intraspinal interventions. It is important to verify that patients are not on medications that would interfere with bleeding and clotting. The patient's pain may be related to an infectious process. Therefore, it is recommended that careful screening be carried out to rule out infection. An infectious disease consult may be warranted in some cases. Furthermore, inadvertent procedural trauma to segmental nerve roots by needles can give rise to painful neuropathies. A complica- tion from the placement of a needle or catheter with overpressurization can include perineal numbness, which will usually resolve in 1 to 2 months. Some patients will present with ongoing neuropathological processes affecting the bladder and bowel. Appropriate urodynamic studies may be required. Patients with previous surgery may have a partial cut through the dura, with incomplete healing. The injection of fluids under pressure can readily find a path of least resistance through the dural tear into the subdural space. In patients who have constrictive scar tissue in the epidural space as well as arachnoiditis, even a small volume of subdural injection of any substance can give rise to significant subsequent deficits as evidenced by bowel, bladder, and sexual dysfunction and weakness/ numbness spreading to greater than anticipated dermatomal areas.

The results of this case study show that ventral epidural lysis of adhesions with hypertonic saline neurolysis is effective in managing chronic low back and lower extremity pain in patients who failed to respond to other conservative modalities of treatment, including fluoroscopically directed transforaminal epidural steroid injections. This study showed that significant pain relief was seen in almost all patients; even though at 6 months only $27 \%$ of the patients experienced relief, which declined to $20 \%$ at 1 year. In addition, the results of the present study are not superior to the controlled studies with application of caudal epidural adhesiolysis $(5,6,13)$.

This case analysis is the first report of treatment of patients with transforaminal epidural adhesiolysis. However, the study was neither prospective nor randomized. Hence, this report may be criticized for its retrospective nature. However, we believe that the results are promising; and a controlled, prospective, randomized clinical trial is essential to prove these results. It may also be worthwhile to compare the results of transforaminal ventral epidural adhesiolysis with caudal or interlaminar epidural adhesiolysis.

\section{CONCLUSION}

Transforaminal epidural adhesiolysis is proposed as a novel therapeutic intervention to treat low back and leg pain resulting from epidural adhesions. The recommended approach to treating radicular pain from L1-5 would be transforaminal, whereas a caudal approach is more appropriate at the S1 level. Although further controlled studies are warranted, transforaminal ventral epidural adhesiolysis appears to offer a promising alternative method of treating 
intractable low back pain and radicular pain in patients with failed back surgery.

\section{REFERENCES}

1. North RB, Campbell JN, James CS et al. Failed back surgery syndrome: 5 year follow-up in 102 patients undergoing repeated operation. Neurosurgery 1991; 28:685-691.

2. McCarron RF. Epidural fibrosis: Experimental model and therapeutic alternatives. In Racz GB (ed). Techniques of Neurolysis. Kluwer Academic Publishers, Boston, 1989, pp 87-94.

3. Racz GB, Holubec JT. Lysis of adhesions in the epidural space. In Racz GB (ed). Techniques of Neurolysis. Kluwer Academic Publishers, Boston, 1989, pp 57-72.

4. Cook SD, Prewett AB, Dalton JE et al. Reduction in perineural scar formation after laminectomy with Polyactive ${ }^{\circledR}$ membrane sheets. Spine 1994; 19:18151825.

5. Racz GB, Heavner JE, Raj PP. Percutaneous epidural neuroplasty. Prospective one-year follow up. Pain Digest 1999; 9:97-102.

6. Heavner JE, Racz GB, Raj P. Percutaneous epidural neuroplasty. Prospective evaluation of $0.9 \% \mathrm{NaCl}$ versus $10 \% \mathrm{NaCl}$ with or without hyaluronidase. Reg Anesth Pain Med 1999; 24:202-207.

7. Manchikanti L, Pakanati RR, Bakhit CE et al. Role of adhesiolysis and hypertonic saline neurolysis in management of low back pain. Evaluation of modification of Racz Protocol. Pain Digest 1999; 9:9196.

8. Manchikanti L, Bakhit CE. Percutaneous lysis of epidural adhesions. Pain Physician 2000; 3:46-64.

9. Manchikanti L, Singh V, Kloth D et al. Interventional techniques in the management of chronic pain: Part 2.0. Pain Physician 2001; 4: 24-96.

10. Wilkinson HA. Introduction: Etiology, diagnosis, and therapy. In The Failed Back Syndrome. Etiology and Therapy, Second Edition. Springer-Verlag, New York, 1992, pp 1-3.

11. Waddell G, Kummel EG, Lotto WN et al. Failed lumbar disc surgery and repeat surgery following industrial injury. J Bone Joint Surg (Am) 1979; 61:201-207.

12. Fritsch EW, Heisel J, Rupp S. The failed back surgery syndrome. Reasons, intraoperative findings, and longterm results: A report of 182 operative treatments. Spine 1996; 21:626-633.

13. Manchikanti L, Pampati V, Fellows B et al. Role of one day epidural adhesiolysis in management of chronic low back pain: A randomized clinical trial. Pain Physician 2001; 4:153-166.

14. Annertz M, Jonsson B, Stromqvist B et al. No relationship between epidural fibrosis and sciatica in the lumbar post disc syndrome: A study with contrast enhanced MRI imaging in somatic and asymptomatic patients. Spine 1995; 20:449-453.

15. Cervellini P, Curri D, Volpin L et al. Computed tomography of epidural fibrosis after discectomy: A comparison between symptomatic and asymptomatic patients. Neurosurgery 1998; 23:710-713.

16. Anderson S. A rationale for the treatment algorithm of failed back surgery syndrome. Cur Rev Pain 2000; 4:395-406.

17. Pawl RP. Arachnoiditis and epidural fibrosis: The relationship to chronic pain. Cur Rev Pain 1998; 2:9399.

18. Anderson SR, Racz GB, Heavner J. Evolution of epidural lysis of adhesions. Pain Physician 2000; 3:262270.

19. Barsa JE, Charlton JE. Diagnosis of epidural scarring and its possible contribution to chronic low back pain syndrome. Pain 1984; S4:376.

20. Songer MH, Ghosh L, Spencer DL. Effects of sodium hyaluronate on peridural fibrosis after lumbar laminectomy and discectomy. Spine 1990; 15:550-554.

21. Kuslich SD, Ulstrom CL, Michael CJ. The tissue origin of low back pain and sciatica. Orthop Clin North Am 1991; 22:181-187.

22. Key JA, Ford LT. Experimental intervertebral disc lesions. J Bone Joint Surg Am 1948; 30A:621-630.

23. Robechhi A., Capra R. L'idrocortisone (composto F). Prime esperienze cliniche in campo reumatologico. Minerva Med 1952; 98:1259-1263.

24. Lievre JA, Block-Michel H, Attali P. L'injection transscree etude clinique et radiologrique. Bull Soc Med Hop 1957; 73:1110-1118.

25. Arthur J, Racz G, Heinnrich R et al. Epidural space. Identification of filling defects in lysis of adhesions in the treatment of chronic painful conditions. In Proceedings of the $7^{\text {th }}$ World Congress of Pain. Paris, IASP Publications, 1993, pp 557.

26. Manchikanti L, Pakanati RR, Pampati V. Comparison of three routes of epidural steroid injections in low back pain. Pain Digest 1999; 9:277-285.

27. Manchikanti L. Transforaminal lumbar epidural steroid injections. Pain Physician 2000; 3:374-398.

28. Saal JS, Saal JA. Comprehensive cervical and lumbar intra-spinal injection course. Stanford University School of Medicine, Stanford, CA, July 11-12, 1998.

29. Andrade A, Eckman E. The distribution of radiologic contrast media by lumbar translaminar and selective neural canals in normal human volunteers. Proceedings of the Annual Meeting of the International Spinal Injection Society. Keystone, CO, January 1992.

30. Long DM. Reoperation on the lumbar spine. In Long DM, McAfee PC (eds). Atlas of Spine Surgery. William and Wilkins, Baltimore, 1992, pp 23-57.

31. Marshall LL, Trethewie ER. Chemical irritation of nerve root in prolapsed disc. Lancet 1973; 2:320.

32. Pennington JB, McCarron RF, Laros GS. Identifica- 
tion of ISG in the canine intervertebral disc. Spine 1988; 13:909-912.

33. Saal JS, Franson RC, Dobrow R et al. High levels of inflammatory phospholipase A2 activity in lumbar disc herniations. Spine 1990; 14:674-678.

34. Naylor A, Happey F, Turner RL et al. Enzymatic and emulogical activity in the intervertebral disc. Orthop Clin North Am 1975; 6:51-58.

35. Olmarker K, Rydevik B, Nordborg C. Autologous nucleus pulposus induces neurophsysiological and histological changes in the porcine cauda equina nerve roots. Spine 1993; 18:1425-1432.

36. Cautico W, Parker JC, Pappert E et al. An anatomical and clinical investigation of spinal meningeal nerves. Journal AetaNeurochir (Wien) 1988; 90:139-143.

37. Imai $\mathrm{S}$, Hukudo $\mathrm{T}$, Toshihiro $\mathrm{M}$ et al. Innervating nociceptive networks in the rat lumbar posterior longitudinal ligaments. Spine 1995; 20:2086-2092.

38. Nakamura A, Takahashi K, Takahashi Y et al. Origin of nerve supply in the posterior portion of the lumbar intervertebral disc in rat. Spine 1996; 20:917-924.

39. Hause M, Kikuchi S, Sakuyama Y et al. Autonomic study of the interrelationship between lumbar sacral nerve roots and their surrounding tissue. Spine 1983; 8:50-58.

40. Spencer DL, Irwin GS, Miller JA. Anatomy and significance of fixation of lumbosacral nerve roots in sciatica. Spine 1983; 8:672-679.

41. Hasegawa T, Mikawa $\mathrm{Y}$, Watanabe $\mathrm{R}$ et al. Morphometric analysis of the lumbosacral roots and dorsal root ganglia by magnetic resonance imaging. Spine 1986; 21:1005-1009.

42. Luyendijk W, Van Voortheisen AE. Contrast examination of the spinal epidural space. Acta Radiol 1996; 5:1055-1066.

43. Blomberg R. A method of epiduroscopy and spinaloscopy: Presentation of preliminary results. Acta Anaesthesiol Scand 1985; 29:113-116.

44. Blomberg R. The dorsal median connective tissue band in the lumbo epidural space in humans: An anatomical model using epiduroscopy in autopsy cases. Anesth Analg 1996; 65:217-220.

45. Savolaine ER, Pandya JB, Greenblatt SH et al. Anatomy of the human lumbar epidural space: New insights using CT-epidurography. Anesthesiology 1988; 68:217-220. 